\title{
Vies médiévales de Marie-Madeleine. Introduction, édition du corpus, présentations, notes et annexes par Olivier Collet et Sylviane Messerli
}

\section{Barbara Ferrari}

\section{(2) OpenEdition}

\section{Journals}

\section{Edizione digitale}

URL: http://journals.openedition.org/studifrancesi/5532

DOI: 10.4000/studifrancesi.5532

ISSN: 2421-5856

\section{Editore}

Rosenberg \& Sellier

\section{Edizione cartacea}

Data di pubblicazione: 1 septembre 2011

Paginazione: 385-386

ISSN: 0039-2944

\section{Notizia bibliografica digitale}

Barbara Ferrari, «Vies médiévales de Marie-Madeleine. Introduction, édition du corpus, présentations, notes et annexes par Olivier Collet et Sylviane Messerli», Studi Francesi [Online], 164 (LV | II) | 2011, online dal 30 novembre 2015, consultato il 12 janvier 2021. URL: http://journals.openedition.org/ studifrancesi/5532 ; DOI: https://doi.org/10.4000/studifrancesi.5532

Questo documento è stato generato automaticamente il 12 janvier 2021.

\section{c)}

Studi Francesi è distribuita con Licenza Creative Commons Attribuzione - Non commerciale - Non opere derivate 4.0 Internazionale. 


\title{
Vies médiévales de Marie-Madeleine. Introduction, édition du corpus, présentations, notes et annexes par Olivier Collet et Sylviane Messerli
}

\author{
Barbara Ferrari
}

\section{NOTIZIA}

Vies médiévales de Marie-Madeleine. Introduction, édition du corpus, présentations, notes et annexes par Olivier CoLLET et Sylviane MESSERLI, Turnhout, Brepols, 2008 («Textes vernaculaires du Moyen Âge», 3), 709 pp.

1 Il personaggio della Maddalena, nel quale confluiscono i tratti di diverse figure evangeliche femminili, non ha mai cessato di nutrire l'immaginario cristiano e ha conosciuto nel Medioevo una grandissima popolarità; ciononostante, il ricco dossier delle vite francesi medievali restava solo in parte accessibile, soprattutto per quanto riguarda le versioni in prosa. Grazie al volume di Olivier Collet e Sylviane Messerli, abbiamo ora accesso a una trentina di vite, ciascuna pubblicata tenendo conto dell'intera tradizione manoscritta, il che ha portato gli editori ad esaminare, direttamente nella maggior parte dei casi, più di un centinaio di codici. Come essi stessi sottolineano (p. 7), il loro lavoro non avrebbe potuto conseguire tali risultati senza l'apporto fondamentale dell'Institut de Recherche et d'Histoire des Textes (IRHT), e in particolare della Section romane, la cui ricchissima documentazione relativa all'agiografia francese medievale sta confluendo nella base Jonas, già disponibile on-line (jonas.irht.cnrs.fr), strumento ormai indispensabile per la ricerca in ambito agiografico. Come ricordato nell'introduzione (pp. 9-38), la presentazione quasi integrale del dossier delle vite francesi della santa (cfr. alle pp. 21-23 i criteri di delimitazione del corpus), consente di confrontare le diverse tecniche di traduzione e analizzare i procedimenti di riscrittura; offre inoltre materiali preziosi alle ricerche linguistiche, ma anche 
all'interpretazione letteraria. Questo lavoro va peraltro ad arricchire le numerose edizioni di vite della Maddalena redatte in diverse lingue, testimonianza di un interesse generale per una materia che appartiene a pieno titolo alla storia della trasmissione culturale in Europa (per il provenzale, oltre ai lavori ottocenteschi dello Chabaneau, va ricordata l'edizione di M.C. Marinoni, Il poemetto occitanico sulla 'Vita di Maria Maddalena'. Edizione critica, Milano, CUEM, 2002).

2 Lo studio delle fonti, condotto per ciascuna delle vite pubblicate (quasi tutte in prosa a parte tre poemi e due frammenti), evidenzia nella quasi totalità dei casi la derivazione diretta o indiretta da testi latini, tra i quali spicca, come era inevitabile, la Legenda aurea, all'origine di ben 14 versioni. I leggendari, domenicani e non, svolgono senz'altro un ruolo essenziale nella trasmissione dei modelli delle vite francesi della Maddalena, e sono in primo piano anche nella diffusione di queste; la grande maggioranza delle versioni in prosa non hanno infatti una circolazione indipendente ma sono inserite nelle grandi raccolte vernacolari costituitesi tra il xIII e il xv secolo.

Alcune prudenti indicazioni sui possibili destinatari, sulla provenienza e sulla datazione del corpus sono seguite da una sintetica presentazione di come il personaggio della santa è venuto a formarsi nella tradizione patristica, che assimila alla Maria di Magdala liberata da sette demoni (colei che è accanto a Maria ai piedi della croce e per prima porta agli apostoli l'annuncio della resurrezione) l'anonima peccatrice di Luca 7 e Maria di Betania, sorella di Marta e di Lazzaro. Gli episodi della vita leggendaria creata dagli agiografi medievali sono brevemente richiamati, con particolare attenzione al "miracolo di Marsiglia", presente in tutte le versioni vernacolari, ma per il quale l'identificazione delle fonti resta ancora problematica; non sarebbe infatti sufficientemente fondato l'accostamento alla storia di Apollonio di Tiro avanzato da alcuni studiosi. Alcune pagine sono dedicate alla presentazione del corpus, della sua organizzazione e dei principi editoriali seguiti nella pubblicazione dei testi. Un'ampia bibliografia ragionata (pp. 27-38) conclude l'introduzione, cui fa seguito l'Inventaire $d u$ corpus et de sa tradition manuscrite, con l'elenco delle 28 versioni e dei codici che le conservano (pp. 39-44).

4 La seconda parte del volume («Vies médiévales de Marie-Madeleine. Textes et présentations», pp.45-650) è dedicata all'edizione dei testi, organizzati in quattro gruppi: il primo comprende le versioni la cui fonte è anteriore a Jacopo da Varazze (9), il secondo, il più nutrito, le traduzioni dalla Legenda aurea (14); seguono le compilazioni da fonti diverse (3) e le versioni che sono pervenute in stato frammentario (2). Ciascuna edizione è preceduta da una ricca introduzione in cui vengono prese in esame: la tradizione manoscritta, la o le fonti identificabili, la composizione e le caratteristiche letterarie delle diverse redazioni, gli eventuali problemi filologici. Le osservazioni linguistiche riguardano quasi esclusivamente l'aspetto lessicale, con particolare attenzione ai regionalismi e ai termini che possono fornire indicazioni sulla cronologia dei testi. Per quanto riguarda l'aspetto ecdotico, come dichiarato nell'introduzione, la pratica editoriale adottata non mira alla «reconstitution d'un état idéal de chaque texte» (p. 24). Anche nelle tradizioni non unitestimoniali viene pubblicato il testo di un manoscritto, corretto solo in caso di errori flagranti. L'apparato a piè di pagina, oltre $a$ rendere conto dell'aspetto paleografico del ms. base e a registrarne le lezioni rigettate, riporta comunque in una seconda fascia le varianti significative di tutti gli altri manoscritti. 
5 Non è certo possibile soffermarsi su ciascuna versione, ci limiteremo quindi a sottolineare il valore di un lavoro molto accurato e sempre di prima mano, che anche per testi già pubblicati (come è il caso ad esempio della prima vita attestata: Nantes, Musée Dobrée 5, XIII in.), propone edizioni originali. Naturalmente, in una così vasta ricerca è sempre possibile riscontrare qualche svista o omissione senza che questo metta in discussione la rilevanza dei risultati conseguiti. Segnaliamo, ad esempio, che tra le leggende in versi del ms. Bruxelles, Bibl. Royale de Belgique 10295-304 (versione 3), non figura la vita della prostituta pentita santa Maria Egiziaca, bensì quella della vergine santa Marina (p. 113); nel capitolo dedicato alla vita tramandata dal ms. Tours, BM 1008 (versione 12, pp. 339-344), non si fa menzione degli studi di Fabrizio Cigni (in Poeti e poesia a Genova (e dintorni), Alessandria 2006 e in «Studi mediolatini e Volgari», 51, 2005) che localizzano il codice nell'area ligure genovese.

6 La parte finale del prezioso volume è ancora arricchita da un capitolo dedicato alle illustrazioni dei manoscritti studiati («Iconographie», pp.651-658), da una lista ragionata dei codici utilizzati o citati (pp. 659-664), da un utilissimo «Tableaux récapitulatifs du corpus et de sa tradition manuscrite» (pp. 665-696) e da due indici: delle parole oggetto di commento (pp. 697-701) e delle citazioni bibliche (pp. 703-704). 